\title{
RELIABLE STATE-SPACE UPPER BOUNDS FOR THE PEAK STRUCTURED SINGULAR VALUE ${ }^{\dagger}$
}

\author{
ANDREW G. SPARKS ${ }^{1 *}$ AND DENNIS S. BERNSTEIN ${ }^{2}$ \\ ${ }^{1}$ USAF Wright Laboratory, WL/FIGC, 2210 Eighth Street, Suite 21, Wright Patterson AFB, OH 45433-7531, U.S.A. \\ ${ }^{2}$ Department of Aerospace Engineering, The University of Michigan, Ann Arbor, MI 48109-2118, U.S.A.
}

\begin{abstract}
SUMMARY
A state-space method for computing upper bounds for the peak of the structured singular value over frequency for both real and complex uncertainties is presented. These bounds are based on the positivity and Popov criteria for one-sided, sector-bounded and for norm-bounded, block-structured linear uncertainty. These criteria are restated and used to derive upper bounds for the peak structured singular value by equating the feasibility of a linear matrix inequality which involves a plant state-space realization to the strict positive realness of a transfer function. Numerical examples are given to illustrate these upper bounds. (C) 1998 John \& Wiley Sons, Ltd.
\end{abstract}

Key words: robustness; structured singular value; Popov criterion

\section{INTRODUCTION}

Computation of the structured singular value for robust stability analysis is an intractable problem. Hence, upper bounds for the structured singular value are used in practice. In particular, the upper bounds of Reference 1 for complex uncertainty and Reference 2 for mixed real and complex uncertainty are stated in terms of the frequency response of the plant transfer function, and hence are evaluated at a finite set of frequencies.

Parametric model uncertainty is frequency dependent, while the frequency-dependent component of unstructured model uncertainty can be absorbed into the plant model. Hence, the reciprocal of the peak value of these upper bounds over frequency provides a lower bound for the size of the smallest destabilizing perturbation. Thus, robustness is characterized by the peak of these upper bounds.

This paper was recommended for publication by editor $V$. Balakrishnan

\footnotetext{
*Correspondence to: Andrew G. Sparks, USAF Wright Laboratory, WL/FIGC, 2210 Eighth Street, Suite 21, Wright Patterson AFB, OH 45433-7531, U.S.A.

${ }^{\dagger}$ This paper was originally submitted for consideration in the LMI Special Issue (Volume 6, Number 9/10, November-December 1996); the extensive revisions suggested by the reviewers precluded its inclusion in the Special Issue.
}

Contract grant sponsor: Air Force Office of Scientific Research

Contract grant number: F49620-92-J-0127.

CCC 1049-8923/98/070611-20\$17.50

(C) 1998 John Wiley \& Sons, Ltd. 
In practice, it is often difficult to reliably compute the peak value of the upper bound over frequency. For example, since plants with lightly damped modes have poles close to the imaginary axis, a small perturbation can destabilize the system. Not surprisingly, it is often the case that upper bounds for the structured singular value have sharp peaks. Since the grid of frequencies near the peak must be dense, computing these upper bounds may be difficult to do reliably.

In the case of real parameter uncertainty, the structured singular value may be discontinuous, so that this problem may be more severe. One approach to this problem involves adding a small amount of phase uncertainty to each uncertain real parameter. ${ }^{3}$ However, the phase uncertainty increases the conservatism of the upper bound, and the essential problem of a sharp peak would be likely to remain.

To demonstrate the potential difficulty with finding the size of the smallest destabilizing perturbation using upper bounds, consider the nominal plant model

$$
G(s)=\frac{-0 \cdot 25 s+1}{3 s^{2}+s+3}
$$

in a negative feedback interconnection with a real uncertain parameter $\delta$. Using the closed-loop characteristic equation $3 s^{2}+(1-0 \cdot 25 \delta) s+(3+\delta)=0$ yields

$$
\mu(G(j \omega))= \begin{cases}\frac{1}{3} & \text { if } \omega=0 \\ \frac{1}{4} & \text { if } \omega=\sqrt{\frac{7}{3}} \\ 0 & \text { otherwise }\end{cases}
$$

while the upper bound of Reference 2 for real uncertainty yields $\mu(G(j \omega))$ exactly. However, since the frequencies at which $\mu$ is non-zero are not generally known a priori, frequency-dependent upper bounds may not be reliable for determining the size of the smallest destabilizing perturbation for problems with uncertain real parameters. Alternatively, applying the upper bound of Reference 1 for complex uncertainty is conservative, as shown in Figure 1. Note that this is just the Bode magnitude plot of $G(s)$. Hence, the frequency at which the maximum occurs can be computed as approximately $0.9375 \mathrm{rad} / \mathrm{sec}$ and the peak value can be computed as approximately $1 \cdot 0437$.

In Reference 4, frequency domain upper bounds for the structured singular value were derived using the positivity and Popov criteria in the form of positive real tests for transfer functions evaluated at each frequency. In the present paper, these positive real tests are rewritten in the form of linear matrix inequalities (LMIs) that involve a realization of the plant transfer function. State-space upper bounds for the structured singular value are derived using these LMIs and are shown to provide upper bounds for the peak values of the frequency domain upper bounds. This form of the state-space upper bounds is computationally attractive since the solution of each LMI involves a convex optimization problem that can be solved efficiently using interior point methods. ${ }^{5,6}$ Numerical examples are given to show that the state-space upper bounds are upper bounds for the peak values of the corresponding frequency domain upper bounds, so that the need for searching over frequency can be eliminated.

The results here are related to the work of several other researchers. The most noteworthy contribution to the use of multipliers in robustness theory appears in References 7 and 8, where the authors formulate robustness tests using the concept of passivity. In Reference 9, the authors use multiplier theory to formulate a number of robustness tests for different kinds of uncertainty, including real parameter uncertainty. The results presented in this paper are similar to those results, but are more rigorously developed and are set in the context of the peak of the structured 


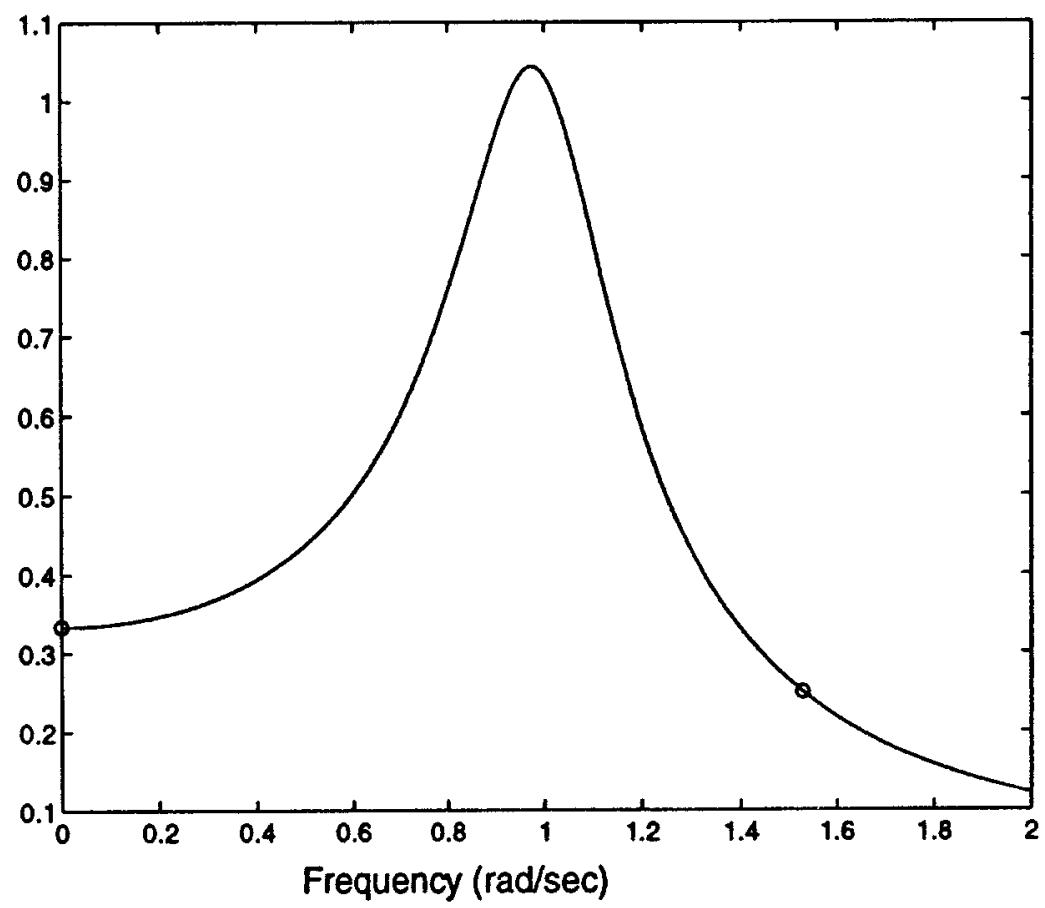

Figure 1. Complex $\mu$ upper bound and real $\mu$ for $G(s)=\frac{-0 \cdot 25 s+1}{3 s^{2}+s+3}$

singular value. The idea of computing the peak of the structured singular value to eliminate a search over frequency is first given in Reference 10, where the peak of the structured singular value for complex uncertainty given in Reference 1 is written. There has been little development of this idea since then, one exception being the results presented in Reference 11, where the peak of the structured singular value for complex uncertainty is formulated as a single optimization problem rather than an optimization problem at each frequency. The results presented here provide a much more straightforward and computationally simple procedure for the peak of the structured singular value, and exploit multiplier theory to reduce the conservatism in the case of real parameter uncertainty.

\section{MATHEMATICAL PRELIMINARIES}

In this section we introduce definitions and present results that will be used in later sections. First we extend the notions of positive realness and strict positive realness to rational functions of a complex variable whose numerator may have complex coefficients. These definitions will be used to state robustness tests in the form of positive real tests that involve complex matrix multipliers and scalings. Next, we extend the positive real lemma to this class of functions and characterize strict positive realness in terms of both a frequency-domain condition on the transfer function and an LMI involving its state-space realization.

Let $Z(s)=C(s I-A)^{-1} B+D$, where $A$ is a real matrix and $B, C$ and $D$ are complex matrices of compatible dimensions. Note that $Z(s)$ is a matrix of rational functions whose denominators have real coefficients and whose numerators have complex coefficients. We shall refer to such functions 
as complex-rational functions. The quadruple $(A, B, C, D)$ is a minimal realization of a complex-rational function if the pair $(A, B)$ is controllable (see Reference 12, Theorem 5-7) and the pair $(A, C)$ is observable (see Reference 12, Theorem 5-13).

Define the paraconjugate transpose of a complex-rational function $Z(s)$ by $Z^{\sim}(s) \triangleq Z^{*}(-\bar{s})$ $=B^{*}\left(-s I-A^{\mathrm{T}}\right)^{-1} C^{*}+D^{*}$. If $Z(s)$ is square, then $H(s) \triangleq Z(s)+Z^{\sim}(s)$ is paraconjugate Hermitian, that is, $H^{*}(s)=H(-\bar{s})$ for all $s \in \mathbb{C}$ (see Reference 13, Definition 2), and thus $H^{*}(j \omega)=H(j \omega)$ is Hermitian for all $\omega \in \mathbb{R}$. Next, a square, real matrix is Hurwitz if the real parts of its eigenvalues are negative. Let the Hermitian part of a square, complex matrix $X$ be denoted by $\mathrm{He} X \triangleq \frac{1}{2}\left(X+X^{*}\right)$, and note that $\mathrm{He} Z(s)=\frac{1}{2}\left(Z(s)+Z^{\sim}(s)\right)$ for all $s=j \omega$. Finally $X>0$ $(X \geqslant 0)$ denotes that the Hermitian matrix $X$ is positive definite (non-negative definite).

\section{Definition 2.1}

An eigenvalue of $A$ is semi-simple if its algebraic multiplicity equals the number of linearly independent eigenvectors associated with it.

\section{Definition 2.2}

A square, complex-rational function $Z(s)=C(s I-A)^{-1} B+D$, where $A \in \mathbb{R}^{n \times n}, B \in \mathbb{C}^{n \times m}$, $C \in \mathbb{C}^{m \times n}$ and $D \in \mathbb{C}^{m \times m}$ are a minimal realization of $Z(s)$, is positive real if $A$ has no eigenvalues in $\operatorname{Re}[s]>0, \operatorname{He} Z(s)>0$ for all $s=j \omega$ such that $j \omega$ is not an eigenvalue of $A$, and every imaginary eigenvalue of $A$ is semi-simple. Furthermore, $Z(s)$ is strictly positive real if there exists $\varepsilon>0$ such that $Z(s-\varepsilon)$ is positive real.

\section{Remark 2.1}

The definition of strict positive realness in the literature varies, the most notable alternative to Definition 2.2 being that $Z(s)$ is strictly positive real if $\mathrm{He} Z(s)>0$ for all $s=j \omega$. As we show later, the definition used in this paper is a sufficient condition for this alternative, and we require our definition for the results in this paper.

\section{Remark 2.2}

If $Z(s)=C(s I-A)^{-1} B+D$ is strictly positive real, then there exists $\varepsilon>0$ such that $Z(s-\varepsilon)=C(s I-(A+\varepsilon I))^{-1} B+D$ is positive real, so that $A+\varepsilon I$ has no eigenvalues in $\operatorname{Re}[s]>0$. Hence $A$ is Hurwitz and $Z(s)$ is asymptotically stable.

The definitions of positive realness and strict positive realness for real-rational functions are motivated from network theory. Every driving point impedance of a passive network is positive real, while every positive real transfer function can be realized as the driving point impedance of a passive network. ${ }^{14}$ Analogously, a strictly positive real transfer function characterizes a dissipative network. To see this, consider a positive real transfer function $Z(s)$ that describes the impedance of a passive network consisting of inductors and capacitors with impedances $s L$ and $1 / s C$. Replacing every term $s L$ by $(s+\varepsilon) L$ (an inductance $L$ in series with resistance $\varepsilon L$ ) and every term $1 / s C$ by $1 /(s+\varepsilon) C$ (a capacitance $C$ in parallel with a resistance $1 / \varepsilon C$ ) yields a dissipative network whose impedance is $Z(s+\varepsilon)$ (see Reference 15, 6, p. 60).

The following result is a generalization of the positive real lemma ${ }^{16,17}$ to positive real and strictly positive real, complex-rational functions. This lemma will be used to relate the frequency domain strict positive real condition to a set of algebraic conditions of a state-space realization. 


\section{Lemma 2.1}

Let $Z(s)=C(s I-A)^{-1} B+D$, where $A \in \mathbb{R}^{n \times n}, B \in \mathbb{C}^{n \times m}, C \in \mathbb{C}^{m \times n}$ and $D \in \mathbb{C}^{m \times m}$ are a minimal realization of $Z(s)$ and $A$ is Hurwitz. Then $Z(s)$ is strictly positive real if and only if there exist a positive-definite matrix $P \in \mathbb{C}^{n \times n}$, matrices $L \in \mathbb{C}^{m \times n}$ and $W \in \mathbb{C}^{m \times m}$, and a positive scalar $\varepsilon$ such that

$$
\begin{aligned}
(A+\varepsilon I)^{\mathrm{T}} P+P(A+\varepsilon I)+L^{*} L & =0 \\
B^{*} P+W^{*} L & =C \\
D+D^{*} & =W^{*} W
\end{aligned}
$$

Proof. Let the positive-definite matrix $P \in \mathbb{C}^{n \times n}$, the matrices $L \in \mathbb{C}^{m \times n}$ and $W \in \mathbb{C}^{m \times n}$, and the positive scalar $\varepsilon$ satisfy (1)-(3). It follows that for all $s \in \mathbb{C}$,

$$
\begin{aligned}
Z(s-\varepsilon)+Z^{*}(s-\varepsilon)= & D+D^{*}+C(s I-(A+\varepsilon I))^{-1} B+B^{*}\left(\bar{s} I-(A+\varepsilon I)^{\mathrm{T}}\right)^{-1} C^{*} \\
= & W^{*} W+W^{*} L(s I-(A+\varepsilon I))^{-1} B+B^{*}\left(\bar{s} I-(A+\varepsilon I)^{\mathrm{T}}\right)^{-1} L^{*} W \\
& +B^{*}\left(\bar{s} I-(A+\varepsilon I)^{\mathrm{T}}\right)^{-1}\left[(\bar{s}+s) P-(A+\varepsilon I)^{\mathrm{T}} P-P(A+\varepsilon I)\right] \\
& \cdot(s I-(A+\varepsilon I))^{-1} B \\
= & W^{*} W+W^{*} L(s I-(A+\varepsilon I))^{-1} B+B^{*}\left(\bar{s} I-(A+\varepsilon I)^{\mathrm{T}}\right)^{-1} L^{*} W \\
& +B^{*}\left(\bar{s} I-(A+\varepsilon I)^{\mathrm{T}}\right)^{-1} L^{*} L(s I-(A+\varepsilon I))^{-1} B \\
& \left.+(\bar{s}+s) B^{*}\left(\bar{s} I-(A+\varepsilon I)^{\mathrm{T}}\right)^{-1} P(s I-(A+\varepsilon I))^{-1} B\right] \\
= & {\left[W+L(s I-(A+\varepsilon I))^{-1} B\right]^{*}\left[W+L(s I-(A+\varepsilon I))^{-1} B\right] } \\
& +(\bar{s}+s) B^{*}(s I-(A+\varepsilon I))^{-*} P(s I-(A+\varepsilon I))^{-1} B
\end{aligned}
$$

so that $Z(s-\varepsilon)+Z^{*}(s-\varepsilon) \geqslant 0$ for all $s=j \omega$. Since $A$ is Hurwitz, we can always choose $\varepsilon>0$ such that $A+\varepsilon I$ has no eigenvalues in $\operatorname{Re}[s]>0$. Hence, there exists $\varepsilon>0$ such that $Z(s-\varepsilon)$ is positive real so that $Z(s)$ is strictly positive real.

Conversely, suppose that $Z(s)$ is strictly positive real so that there exists $\delta>0$ such that $Z(s-\delta)$ is positive real, which implies $Z(s-\varepsilon)$ is positive real for all $\varepsilon \in[0, \delta]$. Hence, since $A$ is Hurwitz, $\varepsilon \in[0, \delta]$ can be chosen such that $Z(s-\varepsilon)$ is positive real and $A+\varepsilon I$ is Hurwitz. Simple manipulations yield the realization

$$
Z(s-\varepsilon)+Z^{\sim}(s-\varepsilon) \sim\left[\begin{array}{cc|c}
A+\varepsilon I & 0 & B \\
0 & -(A+\varepsilon I)^{\mathrm{T}} & C^{*} \\
\hline C & -B^{*} & D+D^{*}
\end{array}\right]=\left[\begin{array}{c|c}
\mathscr{A}_{1} & \mathscr{B}_{1} \\
\hline \mathscr{C}_{1} & \mathscr{D}_{1}
\end{array}\right]
$$

To show that realization (4) is minimal, we first note that since $A$ is Hurwitz, the matrix block-diag $\left(\lambda I-(A+\varepsilon I), \lambda I+(A+\varepsilon I)^{\mathrm{T}}\right)$ has rank $2 n$ for all $\lambda \in \mathbb{C}$ which are not eigenvalues of either $A+\varepsilon I$ or $-(A+\varepsilon I)^{\mathrm{T}}$, so that the matrices

$$
\mathscr{C}=\left[\begin{array}{ccc}
\lambda I-(A+\varepsilon I) & 0 & B \\
0 & \lambda I+(A+\varepsilon I)^{\mathrm{T}} & C^{*}
\end{array}\right], \quad \mathcal{O}=\left[\begin{array}{cc}
\lambda I-(A+\varepsilon I) & 0 \\
0 & \lambda I+(A+\varepsilon I)^{\mathrm{T}} \\
C & -B^{*}
\end{array}\right]
$$

both have rank $2 n$ for all $\lambda \in \mathbb{C}$ which are not eigenvalues of either $A+\varepsilon I$ or $-(A+\varepsilon I)^{\mathrm{T}}$. Next, note that since $(A, B)$ is controllable, $[\lambda I-A B]$ has rank $n$ for all $\lambda \in \mathbb{C}$. It thus follows that 
$[\lambda I-(A+\varepsilon I) B]$ has rank $n$ for all $\lambda \in \mathbb{C}$ so that $\left[{ }^{\lambda I+}+{ }_{-}(A+\varepsilon)^{\mathrm{T}}\right]$ has rank $n$ for all $\lambda \in \mathbb{C}$. Similarly, since $(A, C)$ is observable it follows that $\left[{ }_{C}^{\lambda I-(A+\varepsilon I)}\right]$ has rank $n$ for all $\lambda \in \mathbb{C}$ so that $\left[\lambda I+(A+\varepsilon I)^{\mathrm{T}} C^{*}\right]$ has rank $n$ for all $\lambda \in \mathbb{C}$. Now, $A+\varepsilon I$ has only eigenvalues in the open left-half plane and $-(A+\varepsilon I)^{\mathrm{T}}$ has only eigenvalues in the open right-half plane, so that $A+\varepsilon I$ and $-(A+\varepsilon I)^{\mathrm{T}}$ have no common eigenvalues. Hence, since $\lambda I-(A+\varepsilon I)$ has rank $n$ for all $\lambda \in \mathbb{C}$ which are eigenvalues of $-(A+\varepsilon I)^{\mathrm{T}}$ and $\lambda I+(A+\varepsilon I)^{\mathrm{T}}$ has rank $n$ for all $\lambda \in \mathbb{C}$ which are eigenvalues of $A+\varepsilon I$, the matrices $\mathscr{C}$ and $\mathcal{O}$ both have rank $2 n$ for all $\lambda \in \mathbb{C}$ which are eigenvalues of either $A+\varepsilon I$ or $-(A+\varepsilon I)^{\mathrm{T}}$. Hence the matrices $\mathscr{C}$ and $\mathcal{O}$ both have rank $2 n$ for all $\lambda \in \mathbb{C}$, so that the realization (4) is controllable, and observable, and hence minimal.

Next, since $Z(s-\varepsilon)$ is positive real, it follows from Theorem 2 of Reference 13 that there exists a spectral factorization $Z(s-\varepsilon)+Z^{\sim}(s-\varepsilon)=V^{\sim}(s) V(s)$, where $V(s)=H(s I-F)^{-1} G+J$ is asymptotically stable and $F \in \mathbb{R}^{n \times n}, G \in \mathbb{C}^{n \times m}, H \in \mathbb{C}^{m \times n}$ and $J \in \mathbb{C}^{m \times m}$ are a minimal realization of $V(s)$. Letting $K \in \mathbb{C}^{n \times n}$ satisfy $K F+F^{\mathrm{T}} K+H^{*} H=0$, it follows that

$$
\begin{aligned}
V^{\sim}(s) V(s)= & {\left[G^{*}\left(-s I-F^{\mathrm{T}}\right)^{-1} H^{*}+J^{*}\right]\left[H(s I-F)^{-1} G+J\right] } \\
= & G^{*}\left(-s I-F^{\mathrm{T}}\right)^{-1} H^{*} H(s I-F)^{-1} G+G^{*}\left(-s I-F^{\mathrm{T}}\right)^{-1} H^{*} J \\
& +J^{*} H(s I-F)^{-1} G+J^{*} J \\
= & G^{*}\left(-s I-F^{\mathrm{T}}\right)^{-1}\left(K(s I-F)+\left(-s I-F^{\mathrm{T}}\right) K\right)(s I-F)^{-1} G \\
& +G^{*}\left(-s I-F^{\mathrm{T}}\right)^{-1} H^{*} J+J^{*} H(s I-F)^{-1} G+J^{*} J \\
= & \left(G^{*} K+J^{*} H\right)(s I-F)^{-1} G+G^{*}\left(-s I-F^{\mathrm{T}}\right)^{-1}\left(K G+H^{*} J\right)+J^{*} J
\end{aligned}
$$

Hence a realization of $V^{\sim}(s) V(s)$ is given by

$$
V^{\sim}(s) V(s) \sim\left[\begin{array}{cc|c}
F & 0 & G \\
0 & -F^{\mathrm{T}} & K G+H^{*} J \\
\hline J^{*} H+G^{*} K & -G^{*} & J^{*} J
\end{array}\right]=\left[\begin{array}{c|c}
\mathscr{A}_{2} & \mathscr{B}_{2} \\
\hline \mathscr{C}_{2} & \mathscr{D}_{2}
\end{array}\right]
$$

Since $Z(s-\varepsilon)+Z^{\sim}(s-\varepsilon)=V^{\sim}(s) V(s)$, (4) and (5) are equivalent realizations of the same transfer function. Hence, since (4) is minimal and (4) and (5) are both realizations of order $2 n$, it follows that (5) is also a minimal realization, so that there exists a non-singular matrix $T \in \mathbb{R}^{n \times n}$ such that $\mathscr{A}_{2} T=T \mathscr{A}_{1}, \mathscr{B}_{2}=T \mathscr{A}_{1}, \mathscr{C}_{2} T=\mathscr{C}_{1}$ and $\mathscr{D}_{2}=\mathscr{D}_{1}$.

Next, partition $T$ conformally as $T=\left[\begin{array}{cc}T_{1} & T_{12} \\ T_{21} & T_{2}\end{array}\right]$, so that $\mathscr{A}_{2} T=T \mathscr{A}_{1}$ implies $0=F T_{12}+T_{12}(A+\varepsilon I)^{\mathrm{T}}$. Since $F$ and $A+\varepsilon I$ are Hurwitz, this equation has the unique solution $T_{12}=0$. Similarly, $T_{21}=0$. Hence, $F=T_{1}(A+\varepsilon I) T_{1}^{-1}, G=T_{1} B, J^{*} H+G^{*} K=C T_{1}^{-1}$ and $J^{*} J=D^{*}+D$. Now, define $P \triangleq T_{1}^{\mathrm{T}} K T_{1}, L \triangleq H T_{1}$ and $W \triangleq J$. Then, it can be seen that $P, L$ and $W$ satisfy (1)-(3). Finally, since $T_{1}$ is non-singular and $K>0$, it follows that $P>0$.

The following lemma characterizes strictly positive real complex-rational functions.

\section{Lemma 2.2}

Let $Z(s)=C(s I-A)^{-1} B+D$, where $A \in \mathbb{R}^{n \times n}, B \in \mathbb{R}^{n \times m}, C \in \mathbb{R}^{m \times n}$ and $D \in \mathbb{R}^{m \times m}$ are a minimal realization of $Z(s)$. If $Z(s)$ is strictly positive real, then He $Z(s)>0$ for all $s=j \omega$. Conversely, if $A$ is Hurwitz, $\mathrm{He} Z(s)>0$ for all $s=j \omega$ and $D+D^{*}>0$, then $Z(s)$ is strictly positive real. 
Proof. Suppose that $Z(s)$ is strictly positive real and let $\varepsilon>0$ be such that $Z(s-\varepsilon)$ is positive real. Using Lemma 2.1, it follows from (2) and (3) that

$$
\begin{aligned}
Z(s)+Z^{*}(s)= & D+D^{*}+C(s I-A)^{-1} B+B^{*}\left(\bar{s} I-A^{\mathrm{T}}\right)^{-1} C^{*} \\
= & W^{*} W+\left(W^{*} L+B^{*} P\right)(s I-A)^{-1} B+B^{*}\left(\bar{s} I-A^{\mathrm{T}}\right)^{-1}\left(P B+L^{*} W\right) \\
= & W^{*} W+W^{*} L(s I-A)^{-1} B+B^{*}\left(\tilde{s} I-A^{\mathrm{T}}\right)^{-1} L^{*} W \\
& +B^{*}\left(\bar{s} I-A^{\mathrm{T}}\right)^{-1}\left[(\bar{s}+s) P-A^{\mathrm{T}} P-P A\right]\left(\bar{s} I-A^{\mathrm{T}}\right)^{-1} B
\end{aligned}
$$

Next, using (1) it follows that $A^{\mathrm{T}} P+P A+L^{*} L=-2 \varepsilon P$ so that $L^{*} L<-A^{\mathrm{T}} P-P A$ and

$$
\begin{aligned}
Z(s)+Z^{*}(s)> & W^{*} W+W^{*} L(s I-A)^{-1} B+B^{*}\left(\bar{s} I-A^{\mathrm{T}}\right)^{-1} L^{*} W \\
& +B^{*}\left(\bar{s} I-A^{\mathrm{T}}\right)^{-1} L^{*} L(s I-A)^{-1} B+(\bar{s}+s) B^{*}\left(\bar{s} I-A^{\mathrm{T}}\right)^{-1} P(s I-A)^{-1} B \\
= & {\left[W+L(s I-A)^{-1} B\right]^{*}\left[W+L(s I-A)^{-1} B\right]+(\bar{s}+s) B^{*}\left(s I-A^{\mathrm{T}}\right)^{-*} } \\
& \times P(s I-A)^{-1}
\end{aligned}
$$

Hence, $\mathrm{He} Z(s)>0$ for all $s=j \omega$.

Conversely, suppose $A$ is Hurwitz, He $Z(s)>0$ for all $s=j \omega$ and $D+D^{*}>0$. Let $\varepsilon>0$ and note that

$$
\begin{aligned}
Z(s-\varepsilon) & =C((s-\varepsilon) I-A)^{-1} B+D \\
& =C(s I-A)(s I-(A+\varepsilon I))^{-1}(s I-A)^{-1} B+D \\
& =C(s I-(A+\varepsilon I)+\varepsilon I)(s I-(A+\varepsilon I))^{-1}(s I-A)^{-1} B+D \\
& =Z(s)+\varepsilon Y(s, \varepsilon)
\end{aligned}
$$

where $Y(s, \varepsilon) \triangleq C(s I-(A+\varepsilon I))^{-1}(s I-A)^{-1} B$. Since $A$ is Hurwitz, $s I-A$ is non-singular for all $s=j \omega$ and there exists $\hat{\varepsilon}$ such that $s I-(A+\varepsilon I)$ is non-singular for all $\varepsilon \in[0, \hat{\varepsilon}]$ and for all $s=j \omega$. Hence, $f(\omega, \varepsilon) \triangleq \max _{i}\left|\lambda_{i}[\mathrm{He} Y(j \omega, \varepsilon)]\right|$ is finite for all $\varepsilon \in[0, \hat{\varepsilon}]$ and for all $\omega \in \mathbb{R}$. Since $\lim _{\omega \rightarrow \infty} f(\omega, \varepsilon)=0$ for all $\varepsilon \in[0, \hat{\varepsilon}]$, it follows that there exists $k_{1}>0$ such that $f(\omega, \varepsilon)<k_{1}$ for all $\varepsilon \in[0, \hat{\varepsilon}]$ and for all $\omega \in \mathbb{R}$, so that $-k_{1} I<\operatorname{He} Y(s, \varepsilon)<k_{1} I$ for all $\varepsilon \in[0, \hat{\varepsilon}]$ and for all $s=j \omega$.

Next note that since $\operatorname{He} Z(j \omega)>0$ for all $\omega \in \mathbb{R}$ and $\lim _{\omega \rightarrow \infty} \operatorname{He} Z(j \omega)=D+D^{*}>0$, it follows that there exists $k_{2}>0$ such that $\operatorname{He} Z(s)>k_{2} I$ for all $s=j \omega$. Now, letting $0<\varepsilon<\min \left\{\hat{\varepsilon}, k_{2} / k_{1}\right\}$, we obtain

$$
\text { He } Z(s-\varepsilon)=\operatorname{He} Z(s)+\varepsilon \operatorname{He} Y(s, \varepsilon)>k_{2} I-\varepsilon k_{1} I>0
$$

for all $s=j \omega$. Hence, $Z(s-\varepsilon)$ is positive real so that $Z(s)$ is strictly positive real.

\section{Remark 2.3}

Although the conditions $\mathrm{He} Z(s)>0$ for all $s=j \omega$ and $D+D^{*}>0$ are sufficient for $Z(s)$ to be strictly positive real, the condition $D+D^{*}>0$ is not necessary. For example, consider ${ }^{14}$

$$
Z(s)=\frac{1}{s+\alpha}
$$

where $\alpha>0$, which is clearly strictly positive real, but does not satisfy $D+D^{*}>0$. On the other hand, the condition $\mathrm{He} Z(s)>0$ for all $s=j \omega$ is not sufficient for $Z(s)$ to be strictly positive real. To see this, consider ${ }^{14}$

$$
Z(s)=\frac{s+\alpha+\beta}{(s+\alpha)(s+\beta)}
$$


where $\alpha, \beta>0$. Noting that

$$
\text { He } Z(j \omega)=\frac{\alpha \beta(\alpha+\beta)}{\left(\omega^{2}+\alpha^{2}\right)\left(\omega^{2}+\beta^{2}\right)}
$$

it follows that He $Z(s)>0$ for all $s=j \omega$. Since $Z(s)$ is asymptotically stable, it thus follows from Definition 2.2 that $Z(s)$ is positive real. However, if $\varepsilon>0$ then

$$
\text { He } Z(j \omega-\varepsilon)=\frac{-\varepsilon \omega^{2}+(\alpha-\varepsilon)(\beta-\varepsilon)(\alpha+\beta-\varepsilon)}{\left(\omega^{2}+(\alpha-\varepsilon)^{2}\right)\left(\omega^{2}+(\beta-\varepsilon)^{2}\right)}
$$

is negative for sufficiently large $\omega$, which shows that $Z(s-\varepsilon)$ is not positive real. Hence, although $Z(s)$ satisfies $\mathrm{He} Z(s)>0$ for all $s=j \omega$, it is not strictly positive real.

Finally, the following lemma equates the feasibility of an LMI to the strict positive realness of a complex-rational function.

\section{Lemma 2.3}

Let $Z(s)=C(s I-A)^{-1} B+D$, where $A \in \mathbb{R}^{n \times n}$ is Hurwitz, $B \in \mathbb{C}^{n \times m}, C \in \mathbb{C}^{m \times n}$ and $D \in \mathbb{C}^{m \times m}$ are a minimal realization of $Z(s)$. Then $D+D^{*}>0$ and $Z(s)$ is strictly positive real if and only if there exists a positive-definite matrix $P \in \mathbb{C}^{n \times n}$ such that

$$
\left[\begin{array}{cc}
A^{\mathrm{T}} P+P A & P B-C^{*} \\
B^{*} P-C & -\left(D+D^{*}\right)
\end{array}\right]<0
$$

Proof. Assume that there exists a positive-definite matrix $P \in \mathbb{C}^{n \times n}$ such that (6) holds. Then, letting

$$
F=\left[\begin{array}{cc}
I & 0 \\
\left(D+D^{*}\right)^{-1}\left(B^{*} P-C\right) & I
\end{array}\right] \text { and } G=\left[\begin{array}{cc}
A^{\mathrm{T}} P+P A & P B-C^{*} \\
B^{*} P-C & -\left(D+D^{*}\right)
\end{array}\right]
$$

it follows from (6) that $F^{*} G F<0$, which implies $D+D^{*}>0$ and

$$
0>A^{\mathrm{T}} P+P A+\left(P B-C^{*}\right)\left(D+D^{*}\right)^{-1}\left(B^{*} P-C\right) \triangleq-R
$$

Next, note that

$$
Z(s)+Z^{\sim}(s)=D+D^{*}+C(s I-A)^{-1} B+B^{*}\left(-s I-A^{\mathrm{T}}\right)^{-1} C^{*}
$$

Let $x \in \mathbb{C}^{m}, x \neq 0$, and suppose $B x=0$. Then $x^{*} B^{*}=0$ and

$$
\begin{aligned}
x^{*}\left(Z(j \omega)+Z^{*}(j \omega)\right) x & =x^{*}\left(D+D^{*}\right) x+x^{*} C(s I-A)^{-1} B x+x^{*} B^{*}\left(-s I-A^{\mathrm{T}}\right)^{-1} C^{*} x \\
& =x^{*}\left(D+D^{*}\right) x \\
& >0
\end{aligned}
$$

Next, suppose $B x \neq 0$. Adding and subtracting $B^{*} P(s I-A)^{-1} B+B^{*}\left(-s I-A^{\mathrm{T}}\right)^{-1} P B$ to (8) and using (7) yields

$$
\begin{aligned}
Z(s)+Z^{\sim}(s)= & D+D^{*}-\left(B^{*} P-C\right)(s I-A)^{-1} B-B^{*}\left(-s I-A^{\mathrm{T}}\right)^{-1}\left(P B-C^{*}\right) \\
& +B^{*} P(s I-A)^{-1} B+B^{*}\left(-s I-A^{\mathrm{T}}\right)^{-1} P B \\
= & D+D^{*}-\left(B^{*} P-C\right)(s I-A)^{-1} B-B^{*}\left(-s I-A^{\mathrm{T}}\right)^{-1}\left(P B-C^{*}\right)
\end{aligned}
$$




$$
\begin{aligned}
& +B^{*}\left(-s I-A^{\mathrm{T}}\right)^{-1}\left[\left(-s I-A^{\mathrm{T}}\right) P+P(s I-A)\right](s I-A)^{-1} B \\
= & D+D^{*}-\left(B^{*} P-C\right)(s I-A)^{-1} B-B^{*}\left(-s I-A^{\mathrm{T}}\right)^{-1}\left(P B-C^{*}\right) \\
& +B^{*}\left(-s I-A^{\mathrm{T}}\right)^{-1}\left[\left(P B-C^{*}\right)\left(D+D^{*}\right)^{-1}\left(B^{*} P-C\right)+R\right](s I-A)^{-1} B \\
= & Y^{\sim}(s)\left(D+D^{*}\right)^{-1} Y(s)+B^{*}\left(-s I-A^{\mathrm{T}}\right)^{-1} R(s I-A)^{-1} B
\end{aligned}
$$

where $Y(s) \triangleq\left(D+D^{*}\right)-\left(B^{*} P-C\right)(s I-A)^{-1} B$. Hence,

$$
Z(j \omega)+Z^{*}(j \omega)=Y^{*}(j \omega)\left(D+D^{*}\right)^{-1} Y(j \omega)+B^{*}\left(-j \omega I-A^{\mathrm{T}}\right)^{-1} R(j \omega I-A)^{-1} B
$$

Now, since $D+D^{*}>0$, it follows that $Y^{*}(j \omega)\left(D+D^{*}\right)^{-1} Y(j \omega) \geqslant 0$ for all $\omega \in \mathbb{R}$. Furthermore, since $R>0$, it follows that $\left(-j \omega I-A^{\mathrm{T}}\right)^{-1} R(j \omega I-A)^{-1}>0$ for all $\omega \in \mathbb{R}$. Consequently,

$$
\begin{aligned}
x^{*}\left(Z(j \omega)+Z^{*}(j \omega)\right) x= & x^{*} Y^{*}(j \omega)\left(D+D^{*}\right)^{-1} Y(j \omega) x \\
& +x^{*} B^{*}\left(-j \omega I-A^{\mathrm{T}}\right)^{-1} R(j \omega I-A)^{-1} B x>0
\end{aligned}
$$

for all $\omega \in \mathbb{R}$, and thus $\operatorname{He} Z(s)>0$ for all $s=j \omega$. It now follows from Lemma 2.2 that $Z(s)$ is strictly positive real.

Conversely, suppose that $D+D^{*}>0$ and $Z(s)$ is strictly positive real. Then by Lemma 2.1, there exists a positive-definite matrix $P \in \mathbb{C}^{n \times n}$, and matrices $L \in \mathbb{C}^{m \times n}$ and $W \in \mathbb{C}^{m \times m}$ such that $A^{\mathrm{T}} P+P A+L^{*} L<0$ and such that (2) and (3) hold. Next note that $\left[I-W\left(W^{*} W\right)^{-1} W^{*}\right]\left[I-W\left(W^{*} W\right)^{-1} W^{*}\right] \geqslant 0$ so that $L^{*} L \geqslant L^{*} W\left(W^{*} W\right)^{-1} W^{*} L$. Hence, it follows that $A^{\mathrm{T}} P+P A+\left(P B-C^{*}\right)\left(D+D^{*}\right)^{-1}\left(B^{*} P-C\right)<0$, which can be rewritten using Schur complements as (6).

\section{POSITIVITY AND POPOV CRITERIA}

In this section we state the multivariable positivity and Popov criteria tests for stability of a nominal plant transfer function in a negative feedback interconnection with a real, symmetric, sector-bounded, uncertain matrix representing parameter uncertainty. The Popov criterion, which is a generalization of the positivity criterion, involves a multiplier which plays a key role in reducing the conservatism of the upper bounds for the structured singular value. The positivity and Popov criteria are first written in the form of positive real tests involving the plant transfer function. Then, using Lemma 2.3, these criteria are rewritten in the form of LMIs involving state-space realization of the plant transfer function.

Consider a square nominal transfer function $G(s)$ as shown in Figure 2 in a negative feedback interconnection with a real, square, symmetric, uncertain matrix $F$ which belongs to the set of sector-bounded matrices

$$
\mathscr{F} \triangleq\left\{F \in \mathbb{R}^{m \times m}: F=F^{\mathrm{T}}, 0 \leqslant F \leqslant M\right\}
$$

where $M \in \mathbb{R}^{m \times m}$ is positive definite. Note that this definition will be used in subsequent sections as well. This type of sector bound is referred to as one-sided to denote the fact that the lower bound is zero.

We also consider the subset $\mathscr{F}_{\text {bs }}$ of $\mathscr{F}$ consisting of real, symmetric, block-structured matrices defined by

$$
\mathscr{F}_{\mathrm{bs}} \triangleq\left\{F \in \mathscr{F}: \mathrm{F}=\text { block-diag }\left(I_{l_{1}} \otimes F_{1}, \ldots, I_{l_{r}} \otimes F_{r}\right), F_{i}=F_{i}^{\mathrm{T}} \in \mathbb{R}^{m_{i} \times m_{i}}, i=1, \ldots, r\right\}
$$

where the dimension $m_{i}$ of each block and the number of repetitions $l_{i}$ of the block $F_{i}$ are given. Note that $m=\sum_{i=1}^{r} l_{i} m_{i}$. 


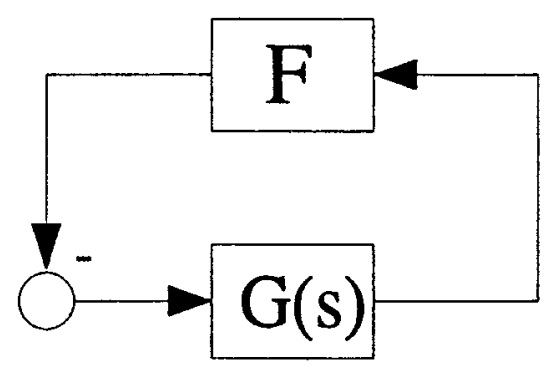

Figure 2. Interconnection of transfer function $G(s)$ with real uncertain matrix $F$

To state the Popov criterion, define the set $\mathscr{N}$ of Hermitian matrices $N$ that commute with every matrix $F \in \mathscr{F}_{\text {bs }}$ by

$$
\mathscr{N} \triangleq\left\{N: N=\text { block-diag }\left(N_{1} \otimes I_{m_{1}}, \ldots, N_{r} \otimes I_{m_{r}}\right), N_{i}=N_{i}^{*} \in \mathbb{C}^{l_{i} \times l_{i}}, i=1, \ldots, r\right\}
$$

Note that if $F \in \mathscr{F}_{\text {bs }}$ and $N \in \mathscr{N}$, then $F N=N F=$ block-diag $\left(N_{1} \otimes F_{1}, \ldots, N_{r} \otimes F_{r}\right)$. We now recall the multivariable positivity and Popov criteria for one-sided, sector-bounded, blockstructured, uncertain matrices.

\section{Lemma $3.1^{18}$}

Let $G(s)$ be an asymptotically stable transfer function. Then the following statements hold.

(i) If $M^{-1}+G(s)$ is strictly positive real, then the negative feedback interconnection of $G(s)$ and $F$ is asymptotically stable for all $F \in \mathscr{F}_{\text {bs }}$.

(ii) If there exists $N \in \mathscr{N}$ such that $M^{-1}+(I+s N) G(s)$ is strictly positive real, then the negative feedback interconnection of $G(s)$ and $F$ is asymptotically stable for all $F \in \mathscr{F}_{\text {bs }}$.

Next we use Lemma 2.3 to rewrite the positivity and Popov criteria from Lemma 3.1 in the form of LMIs that involve a state-space realization of the plant.

\section{Theorem 3.1}

Let $G(s)=C(s I-A)^{-1} B$ be an asymptotically stable transfer function, where $A \in \mathbb{R}^{n \times n}$, $B \in \mathbb{R}^{n \times m}$ and $C \in \mathbb{R}^{m \times n}$. Then the following statements hold.

(i) $M^{-1}+G(s)$ is strictly positive real if and only if there exists a positive-definite matrix $P \in \mathbb{C}^{n \times n}$ such that

$$
\left[\begin{array}{cc}
A^{\mathrm{T}} P+P A & P B-C^{\mathrm{T}} \\
B^{\mathrm{T}} P-C & -2 M^{-1}
\end{array}\right]<0
$$

(ii) Let $N \in \mathscr{N}$. Then $M^{-1}+(I+s N) G(s)$ is strictly positive real if and only if there exists a positive-definite matrix $P \in \mathbb{C}^{n \times n}$ such that

$$
\left[\begin{array}{cc}
A^{\mathrm{T}} P+P A & P B-C^{\mathrm{T}}-A^{\mathrm{T}} C^{\mathrm{T}} N \\
B^{\mathrm{T}} P-C-N C A & -2 M^{-1}-N C B-B^{\mathrm{T}} C^{\mathrm{T}} N
\end{array}\right]<0
$$


In both cases, the negative feedback interconnection of $G(s)$ and $F$ is asymptotically stable for all $F \in \mathscr{F}_{\text {bs }}$.

Proof. First, note that (i) is a special case of (ii) with $N=0$. To prove (ii), note that

$$
M^{-1}+(I+s N) G(s) \sim\left[\begin{array}{c|c}
A & B \\
\hline C+N C A & M^{-1}+N C B
\end{array}\right]
$$

so that $M^{-1}+(I+s N) G(s)$ is an asymptotically stable transfer function. Hence, it follows from Lemma 2.3 with $C$ and $D$ replaced by $C+N C A$ and $M^{-1}+N C B$, respectively, that $M^{-1}+(I+s N) G(s)$ is strictly positive real if and only if there exists a positive-definite matrix $P \in \mathbb{C}^{n \times n}$ such that (10) holds. It now follows from Lemma 3.1 that the negative feedback interconnection of $G(s)$ and $F$ is asymptotically stable for all $F \in \mathscr{F}_{\text {bs }}$.

\section{NORM-BOUNDED, BLOCK-STRUCTURED UNCERTAINTY}

In this section, the positivity and Popov criteria are first stated for a nominal plant transfer function $G(s)$ in a feedback interconnection with a real, norm-bounded, block-structured, uncertain matrix $\Delta$ representing the model uncertainty as shown in Figure 3. Then, these criteria are stated for scaled transfer functions, where the scaling does not affect the stability of the feedback interconnection of $G(s)$ and $\Delta$. Finally, these criteria are rewritten in the form of LMIs, which are used in the following section to obtain state-space upper bounds for the structured singular value.

Define the set $\boldsymbol{\Delta}_{\gamma}$ of norm-bounded, real, block-structured matrices by

$$
\Delta_{\gamma} \triangleq\left\{\Delta \in \Delta_{\text {real }}: \sigma_{\max }(\Delta) \leqslant \gamma^{-1}\right\}
$$

where $\Delta_{\text {real }}$ is the set of real, block-structured matrices given by

$$
\boldsymbol{\Delta}_{\text {real }} \triangleq\left\{\Delta: \Delta=\text { block-diag }\left(I_{l_{1}} \otimes \Delta_{1}, \ldots, I_{l_{r}} \otimes \Delta_{r}\right), \Delta_{i} \in \mathbb{R}^{m_{i} \times m_{i}}, i=1, \ldots, r\right\}
$$

and where the dimension $m_{i}$ and the number of repetitions $l_{i}$ of the uncertain block $\Delta_{i}$ are given. Define the shifted transfer function $G_{\gamma}(s)$ by

$$
G_{\gamma}(s) \triangleq\left[I-\gamma^{-1} G(s)\right]^{-1} G(s)
$$

Note from a simple loop-shifting transformation that the stability of the feedback interconnection of $G(s)$ and $\Delta \in \boldsymbol{\Delta}_{\gamma}$ is equivalent to the stability of the feedback interconnection of the shifted transfer function $G_{\gamma}(s)$ and the shifted uncertainty $F=\Delta+\gamma^{-} I \in \mathscr{F}$, where $M=2 \gamma^{-1} I$. Finally,

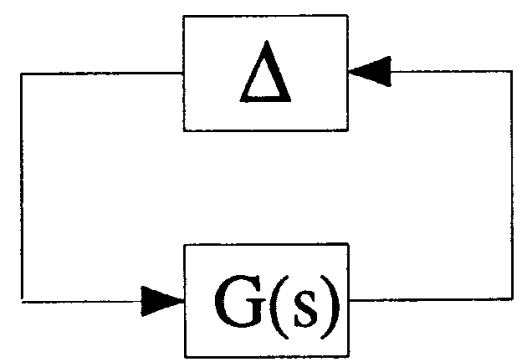

Figure 3. Feedback interconnection of plant $G(s)$ with uncertain matrix $\Delta$ 
define the set $\mathscr{D}$ of complex invertible scaling matrices $D$ that commute with every blockstructured uncertain matrix in $\Delta_{\text {real }}$ by

$$
\mathscr{D} \triangleq\left\{D \in \mathbb{C}^{m \times m}: D=\text { block-diag }\left(D_{1} \otimes I_{m_{1}}, \ldots, D_{r} \otimes I_{m_{r}}\right), D_{i} \in \mathbb{C}^{l_{i} \times l_{i}}, \operatorname{det} D_{i} \neq 0, i=1, \ldots, r\right\}
$$

Note that if $\Delta \in \boldsymbol{\Delta}_{\text {real }}$ and $D \in \mathscr{D}$, then $D \Delta D^{-1}=\Delta D D^{-1}=\Delta$.

We now recall the positivity and Popov criteria and the scaled positivity and scaled Popov criteria for norm-bounded, block-structured uncertain matrices. The positivity and Popov criteria are analogous to the criteria in Lemma 3.1, with $M$ specialized to $2 \gamma^{-1} I$ and the transfer function $G(s)$ replaced by the shifted transfer function $G_{\gamma}(s)$.

\section{Lemma 4.1}

Let $\gamma>0$ and suppose $G_{\gamma}(s)$ is asymptotically stable. Then the following statements hold.

(i) If $\frac{1}{2} \gamma I+G_{\gamma}(s)$ is strictly positive real, then the feedback interconnection of $G(s)$ and $\Delta$ is asymptotically stable for all $\Delta \in \boldsymbol{\Delta}_{\gamma}$.

(ii) If there exists $N \in \mathscr{N}$ such that $\frac{1}{2} \gamma I+(I+s N) G_{\gamma}(s)$ is strictly positive real, then the feedback interconnection of $G(s)$ and $\Delta$ is asymptotically stable for all $\Delta \in \Delta_{\gamma}$.

(iii) If there exists $D \in \mathscr{D}$ such that $\frac{1}{2} \gamma I+D G_{\gamma}(s) D^{-1}$ is strictly positive real, then the feedback interconnection of $G(s)$ and $\Delta$ is asymptotically stable for all $\Delta \in \Delta_{\gamma}$.

(iv) If there exist $D \in \mathscr{D}$ and $N \in \mathscr{N}$ such that $\frac{1}{2} \gamma I+(I+s N) D G_{\gamma}(s) D^{-1}$ is strictly positive real, then the feedback interconnection of $G(s)$ and $\Delta$ is asymptotically stable for all $\Delta \in \Delta_{\gamma}$.

Next, we restate the positivity and Popov criteria in the form of LMIs that involve a state-space realization of the plant transfer function. To do this, define the set $\mathscr{Q}$ of positive-definite matrices $Q$ that commute with every $\Delta \in \Delta_{\text {real }}$ by

$$
\mathscr{Q} \triangleq\{Q \in \mathscr{D}: Q>0\}
$$

Note that if $D \in \mathscr{D}$, then $Q=D^{*} D \in \mathscr{Q}$, while if $Q \in \mathscr{Q}$, the $D=Q^{1 / 2} \in \mathscr{D}$, Note also that if $\hat{N} \in \mathscr{N}$ and $D \in \mathscr{D}$, then $N=D^{*} \hat{N} D \in \mathscr{N}$.

\section{Theorem 4.1}

Let $\gamma>0$ and suppose $G_{\gamma}(s) \sim\left[\frac{A+\gamma^{-1} B C}{C} \mid \frac{B}{0}\right]$ is asymptotically stable, where $A \in \mathbb{R}^{n \times n} B \in \mathbb{R}^{n \times n}$ and $C \in \mathbb{R}^{m \times n}$. Then the following statements hold.

(i) $\frac{1}{2} \gamma I+G_{\gamma}(s)$ is strictly positive real if and only if there exists a positive-definite matrix $P \in \mathbb{C}^{n \times n}$ such that

$$
\left[\begin{array}{cc}
\left(A+\gamma^{-1} B C\right)^{\mathrm{T}} P+P\left(A+\gamma^{-1} B C\right) & P B-C^{\mathrm{T}} \\
B^{\mathrm{T}} P-C & -\gamma I
\end{array}\right]<0
$$

(ii) Let $Q \in \mathscr{Q}$. Then $\frac{1}{2} \gamma I+Q^{1 / 2} G_{\gamma}(s) Q^{-1 / 2}$ is strictly positive real if and only if there exists a positive-definite matrix $P \in \mathbb{C}^{n \times n}$ such that

$$
\left[\begin{array}{cc}
\left(A+\gamma^{-1} B C\right)^{\mathrm{T}} P+P\left(A+\gamma^{-1} B C\right) & P B-C^{\mathrm{T}} Q \\
B^{\mathrm{T}} P-Q C & -\gamma Q
\end{array}\right]<0
$$

(iii) Let $N \in \mathscr{N}$. Then $\frac{1}{2} \gamma I+(I+s N) G_{\gamma}(s)$ is strictly positive real if and only if there exists a positive-definite matrix $P \in \mathbb{C}^{n \times n}$ such that

$$
\left[\begin{array}{cc}
\left(A+\gamma^{-1} B C\right)^{\mathrm{T}} P+P\left(A+\gamma^{-1} B C\right) & P B-C^{\mathrm{T}}-\left(A+\gamma^{-1} B C\right)^{\mathrm{T}} C^{\mathrm{T}} N \\
B^{\mathrm{T}} P-C-N C\left(A+\gamma^{-1} B C\right) & -N C B-B^{\mathrm{T}} C^{\mathrm{T}} N-\gamma I
\end{array}\right]<0
$$


(iv) Let $N \in \mathscr{N}$ and $Q \in \mathscr{Q}$. Then $\frac{1}{2} \gamma I+\left(I+s Q^{-1 / 2} N Q^{-1 / 2}\right) Q^{1 / 2} G_{\gamma}(s) Q^{-1 / 2}$ is strictly positive real if and only if there exists a positive-definite $P \in \mathbb{C}^{m \times n}$ such that

$$
\left[\begin{array}{cc}
\left(A+\gamma^{-1} B C\right)^{\mathrm{T}} P+P\left(A+\gamma^{-1} B C\right) & P B-C^{\mathrm{T}} Q-\left(A+\gamma^{-1} B C\right)^{\mathrm{T}} C^{\mathrm{T}} N \\
B^{\mathrm{T}} P-Q C-N C\left(A+\gamma^{-1} B C\right) & -N C B-B^{\mathrm{T}} C^{\mathrm{T}} N-\gamma Q
\end{array}\right]<0
$$

In all cases, the feedback interconnection of $G(s)$ and $\Delta$ is asymptotically stable for all $\Delta \in \boldsymbol{\Delta}_{\gamma}$.

Proof. First, note that (i)-(ii) are special cases of (iv) with $Q=I$ and/or $N=0$. To prove (iv), define $Z(s) \triangleq \frac{1}{2} \gamma I+\left(I+s Q^{-1 / 2} N Q^{-1 / 2}\right) Q^{1 / 2} G_{\gamma}(s) Q^{-1 / 2}$ and note that

$$
Z(s) \sim\left[\begin{array}{c|c}
A+\gamma^{-1} B C & B Q^{-1 / 2} \\
\hline Q^{1 / 2} C+Q^{-1 / 2} N C\left(A+\gamma^{-1} B C\right) & \frac{1}{2} \gamma I+Q^{-1 / 2} N C B Q^{-1 / 2}
\end{array}\right]
$$

Since $G_{\gamma}(s)$ is asymptotically stable by assumption, it follows that $Z(s)$ is also asymptotically stable. It follows from Lemma 2.3 with $A, B, C$ and $D$ replaced by $A+\gamma^{-1} B C, B Q^{-1 / 2}$, $Q^{1 / 2} C+Q^{-1 / 2} N C\left(A+\gamma^{-1} B C\right)$ and $\frac{1}{2} \gamma I+Q^{-1 / 2} N C B Q^{-1 / 2}$, respectively, that $Z(s)$ is strictly positive real if and only if there exists a positive-definite matrix $P \in \mathbb{C}^{n \times m}$ such that

$$
\left[\begin{array}{cc}
\left(A+\gamma^{-1} B C\right)^{\mathrm{T}} P+P\left(A+\gamma^{-1} B C\right) & P B Q^{-1 / 2}-C^{\mathrm{T}} Q^{1 / 2}-\left(A+\gamma^{-1} B C\right)^{\mathrm{T}} C^{\mathrm{T}} N Q^{-1 / 2} \\
Q^{-1 / 2} B^{\mathrm{T}} P-Q^{1 / 2} C-Q^{-1 / 2} N C\left(A+\gamma^{-1} B C\right) & -Q^{-1 / 2} N C B Q^{-1 / 2}-Q^{-1 / 2} B^{\mathrm{T}} C^{\mathrm{T}} N Q^{-1 / 2}-\gamma I
\end{array}\right]<0
$$

Since $\left[\begin{array}{ll}I & 0 \\ 0 & Q^{1 / 2}\end{array}\right]$ is nonsingular, it follows that (15) is equivalent to (14). Finally, since $Q \in \mathscr{Q}$ and $N \in \mathscr{N}$ imply $Q^{-1 / 2} N Q^{-1 / 2} \in \mathscr{N}$, it follows from Lemma 4.1 that the feedback interconnection of $G(s)$ and $\Delta$ is asymptotically stable for all $\Delta \in \Delta_{\gamma}$.

\section{BOUNDS FOR THE STRUCTURED SINGULAR VALUE}

In this section, we use the positivity and Popov criteria given in the previous section to obtain upper bounds for the structured singular value. Specifically, the positive real criteria are used to obtain upper bounds for the structured singular value in the frequency domain, while the LMI criteria are used to obtain upper bounds for the structured singular value in the time domain. Finally, these time domain bounds are shown to be upper bounds for the peaks of the corresponding frequency domain upper bounds.

Recall that the structured singular value of a complex matrix $G(j \omega)$ for complex, blockstructured uncertainty is defined as ${ }^{1}$

$$
\mu_{\mathrm{com}}(G(j \omega)) \triangleq \frac{1}{\min \left\{\sigma_{\max }(\Delta): \Delta \in \boldsymbol{\Delta}_{\mathrm{com}, 0}\right\}}
$$

where

$$
\boldsymbol{\Delta}_{\text {com }, 0} \triangleq\left\{\Delta \in \boldsymbol{\Delta}_{\text {com }}: \operatorname{det}(I+G(j \omega) \Delta)=0\right\}
$$

and where $\boldsymbol{\Delta}_{\text {com }}$ is the set of complex, block-structured matrices given by

$$
\boldsymbol{\Delta}_{\text {com }} \triangleq\left\{\Delta: \Delta=\text { block-diag }\left(I_{l_{1}} \otimes \Delta_{1}, \ldots, I_{l_{r}} \otimes \Delta_{r}\right), \Delta_{i} \in \mathbb{C}^{m_{i} \times m_{i}}, i=1, \ldots, r\right\}
$$

If $\boldsymbol{\Delta}_{\text {com }, 0}$ is empty, then by convention, $\mu_{\text {com }}(G(j \omega)) \triangleq 0$. Similarly, the structured singular value of $G(j \omega)$ for real, block-structured uncertainty is defined as

$$
\mu_{\text {real }}(G(j \omega)) \triangleq \frac{1}{\min \left\{\sigma_{\max }(\Delta): \Delta \in \Delta_{\text {real }, 0}\right\}}
$$


where

$$
\boldsymbol{\Delta}_{\text {real }, 0} \triangleq\left\{\Delta \in \boldsymbol{\Delta}_{\text {real }}: \operatorname{det}(I+G(j \omega) \Delta)=0\right\}
$$

$\boldsymbol{\Delta}_{\text {real,o }}$ is empty, then $\mu_{\text {real }}(G(j \omega)) \triangleq 0$.

\subsection{Frequency domain bounds}

Using the positivity and Popov criteria, we define $\mu_{\text {pos }}(G(j \omega))$ and $\mu_{\text {Pop }}(G(j \omega))$ by ${ }^{4}$

and

$$
\mu_{\text {pos }}(G(j \omega)) \triangleq \inf \left\{\gamma>0: \operatorname{He}\left[\frac{1}{2} \gamma I+G_{\gamma}(j \omega)\right]>0\right\}
$$

$$
\mu_{\text {Pop }}(G(j \omega)) \triangleq \inf \left\{\gamma>0 \text { : there exists } N \in \mathscr{N} \text { such that } \operatorname{He}\left[\frac{1}{2} \gamma I+(I+j \omega N) G_{\gamma}(j \omega)\right]>0\right\}
$$

Similarly, using the scaled positivity and scaled Popov criteria, we define $\mu_{\mathrm{Pos}, \mathrm{sc}}(G(j \omega))$ and $\mu_{\text {Pop }, \mathrm{sc}}(G(j \omega))$ by

$$
\mu_{\text {Pop }, \mathrm{sc}}(G(j \omega)) \triangleq \inf \left\{\gamma>0 \text { : there exists } D \in \mathscr{D} \text { such that } \operatorname{He}\left[\frac{1}{2} \gamma I+D G_{\gamma}(j \omega) D^{-1}\right]>0\right\}
$$

and

$$
\begin{gathered}
\mu_{\text {Pop ,sc }}(G(j \omega)) \triangleq \inf \{\gamma>0 \text { : there exists } N \in \mathscr{N} \text { and } D \in \mathscr{D} \text { such that } \\
\left.\operatorname{He}\left[\frac{1}{2} \gamma I+(I+j \omega N) D G_{\gamma}(j \omega) D^{-1}\right]>0\right\}
\end{gathered}
$$

In Reference 4, it was shown that $\mu_{\mathrm{pos}}(G(j \omega))$ and $\mu_{\mathrm{pos}, \mathrm{sc}}(G(j \omega))$ are upper bounds for the complex structured singular value, and that $\mu_{\mathrm{Pop}}(G(j \omega))$ and $\mu_{\mathrm{Pop}, \mathrm{sc}}(G(j \omega))$ are bounds for the real structured singular value. Furthermore, these bounds satisfy the ordering

$$
\begin{array}{ccccc}
\mu_{\text {com }}(G(j \omega)) & \leqslant & \mu_{\text {pos }, \mathrm{sc}}(G(j \omega)) & \leqslant & \mu_{\text {pos }}(G(j \omega)) \\
\mathrm{VI} & & \mathrm{VI} & & \mathrm{VI} \\
\mu_{\text {real }}(G(j \omega)) & \leqslant & \mu_{\text {Pop }, \mathrm{sc}}(G(j \omega)) & \leqslant & \mu_{\text {Pop }}(G(j \omega))
\end{array}
$$

\subsection{State-space bounds}

In this section, we present the main result of the paper which provides state-space upper bounds for the structured singular value. These upper bounds are written as LMIs that involve a state-space realization of the plant transfer function. Letting $G(s)=C(s I-A)^{-1} B$, we define

$$
\begin{gathered}
\mu_{\text {Pos,peak }} \triangleq \inf \{\gamma>0: \text { there exists } P>0 \text { such that (11) holds }\} \\
\mu_{\text {Pos,sc,peak }} \triangleq \inf \{\gamma>0: \text { there exists } P>0, Q \in \mathscr{Q} \text { such that (12) holds }\} \\
\mu_{\text {Pop,peak }} \triangleq \inf \{\gamma>0: \text { there exists } P>0, N \in \mathscr{N} \text { such that (13) holds }\} \\
\mu_{\text {Pop,sc,peak }} \triangleq \inf \{\gamma>0: \text { there exists } P>0, N \in \mathscr{N}, Q \in \mathscr{Q} \text { such that (14) holds }\}
\end{gathered}
$$

The following result shows that (22) - (25) are upper bounds for the peak values of the corresponding frequency domain upper bounds.

\section{Theorem 5.1}

$$
\begin{gathered}
\sup _{\omega} \mu_{\text {pos }}(G(j \omega)) \leqslant \mu_{\text {pos,peak }} \\
\sup _{\omega} \mu_{\text {pos,sc }}(G(j \omega)) \leqslant \mu_{\text {pos,sc,peak }}
\end{gathered}
$$




$$
\begin{gathered}
\sup _{\omega} \mu_{\text {Pop }}(G(j \omega)) \leqslant \mu_{\text {Pop,peak }} \\
\sup _{\omega} \mu_{\text {Pop,sc }}(G(j \omega)) \leqslant \mu_{\text {Pop,sc,peak }}
\end{gathered}
$$

Proof. First note that (26) $-(28)$ are special cases of (29) with $Q=I$ and/or $N=0$. To prove (29), note from (25) and Theorem 4.1 that $\mu_{\text {Pop,sc,peak }}$ can be rewritten as

$$
\begin{gathered}
\mu_{\text {Pop,sc,peak }}=\inf \{\gamma>0 \text { : there exists } N \in \mathscr{N} \text { and } Q \in \mathscr{Q} \text { such that } \\
\left.\frac{1}{2} \gamma I+\left(I+s Q^{-1 / 2} N Q^{-1 / 2}\right) Q^{1 / 2} G_{\gamma}(s) Q^{-1 / 2} \text { is strictly positive real }\right\}
\end{gathered}
$$

Since $Q \in \mathscr{Q}$ and $N \in \mathscr{N}$ imply $Q^{-1 / 2} N Q^{-1 / 2} \in \mathscr{N}$ and $Q^{1 / 2} \in \mathscr{D}, \mu_{\text {Pop,sc,peak }}$ can be rewritten

$$
\begin{gathered}
\mu_{\text {Pop,sc,peak }}=\inf \{\gamma>0: \text { there exist } N \in \mathscr{N} \text { and } D \in \mathscr{D} \text { such that } \\
\left.\frac{1}{2} \gamma I+(I+s N) D G_{\gamma}(s) D^{-1} \text { is strictly positive real }\right\}
\end{gathered}
$$

By definition, it follows that for all $\omega \in \mathbb{R}$,

$$
\begin{aligned}
\mu_{\text {Pop }, \text { sc }, \text { peak }} & \geqslant \inf \{\gamma>0: \text { there exist } N \in \mathscr{N} \text { and } D \in \mathscr{D} \text { such that } \\
& \left.\operatorname{He}\left[\frac{1}{2} \gamma I+(I+j \omega N) D G_{\gamma}(j \omega) D^{-1}\right]>0\right\}
\end{aligned}
$$

Hence,

$$
\begin{gathered}
\mu_{\text {Pop,sc,peak }} \geqslant \sup _{\omega} \inf \{\gamma>0: \text { there exist } N \in \mathscr{N} \text { and } D \in \mathscr{D} \text { such that } \\
\left.\operatorname{He}\left[\frac{1}{2} \gamma I+(I+j \omega N) D G_{\gamma}(j \omega) D^{-1}\right]>0\right\}
\end{gathered}
$$

so that, by definition, $\sup _{\omega} \mu_{\text {Pop,sc }}(G(j \omega)) \leqslant \mu_{\text {Pop,sc,peak }}$.

\section{NUMERICAL EXAMPLES}

The state-space bounds (22) - (25) are in the form of LMIs that involve a state-space realization of the plant transfer function. Hence, the state-space bounds can be found by using a bisection algorithm for $\gamma$, where at each step the LMI is solved as a feasibility problem. ${ }^{5,6}$ Thus, at each step, the algorithm reveals whether there exist appropriate matrices such that the LMI is satisfied for a given $\gamma$, and the state-space bounds can be computed to arbitrary accuracy. The size of the smallest destabilizing perturbation is the inverse of the state-space bound.

First, we reconsider the example from Section 1. By evaluating $\mu_{\text {Pop,sc,peak }}$ using a bisection algorithm on $\gamma$, the peak value of the bound for real uncertainty is found to be 0.3334 with an accuracy of $10^{-4}$. The variables $Q$ and $N$ in the LMI are determined to be 0.013 and 0.0243 , respectively. Hence, the state-space bound $\mu_{\text {Pop,sc,peak }}$ was able to accurately compute the size of the smallest destabilizing perturbation efficiently, without an exhaustive search over frequency. The peak upper bound $\mu_{\text {Pop,sc,peak }}$ is shown as the dashed line in Figure 4.

Next, consider the linear plant model whose realization is given by

$$
\begin{gathered}
\dot{x}=\left[\begin{array}{rcc}
-1 & 0 & 0 \\
0 & -0 \cdot 1 & 0.9 \\
0 & -0.9 & -0 \cdot 1
\end{array}\right] x+\left[\begin{array}{ccc}
0.57 & 0.53 & 0.75 \\
0.80 & 0.50 & 0.55 \\
0.03 & 0.96 & 0.89
\end{array}\right] u \\
y=\left[\begin{array}{ccc}
0.62 & 0.21 & 0.09 \\
0.82 & 0.71 & 0.27 \\
0.16 & 0.13 & 0.00
\end{array}\right] x
\end{gathered}
$$




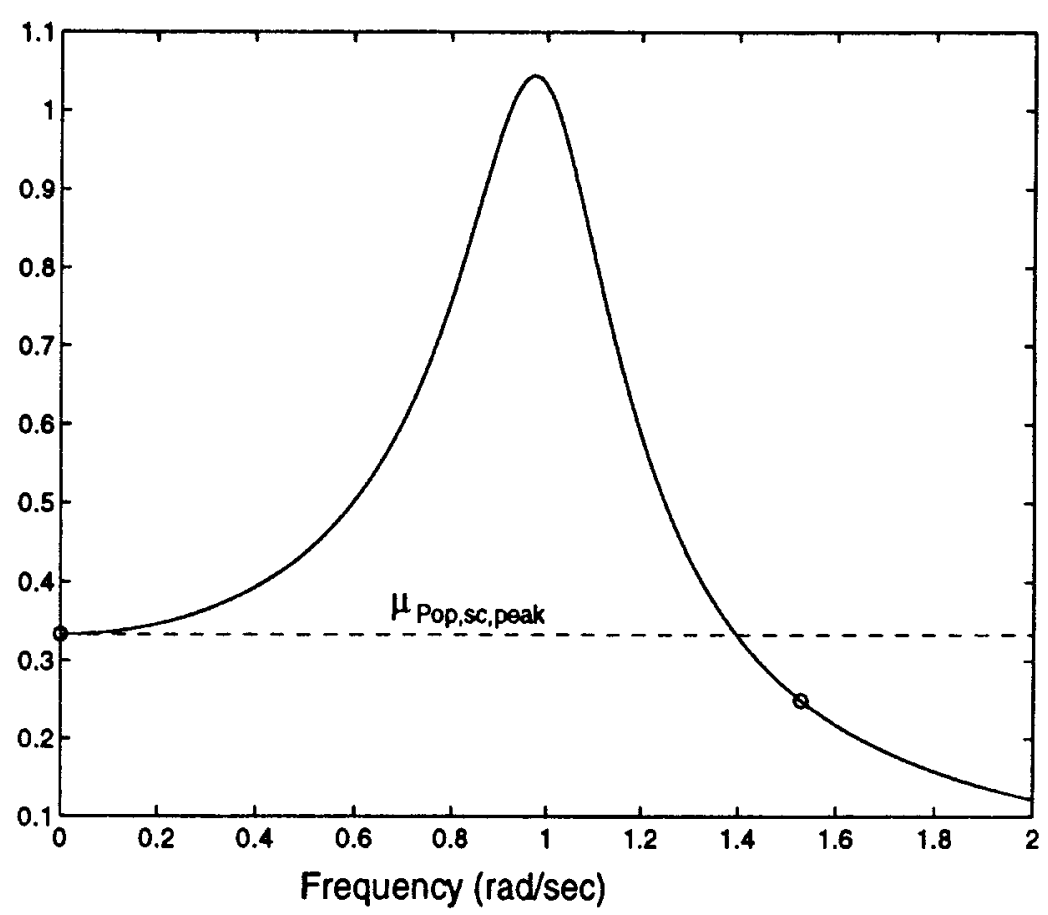

Figure 4. Real $\mu$, complex $\mu$ upper bound and peak upper bound $\mu_{\text {pop,sc,peak }}$ for $\frac{-0 \cdot 25 s+1}{3 s^{2}+s+3}$

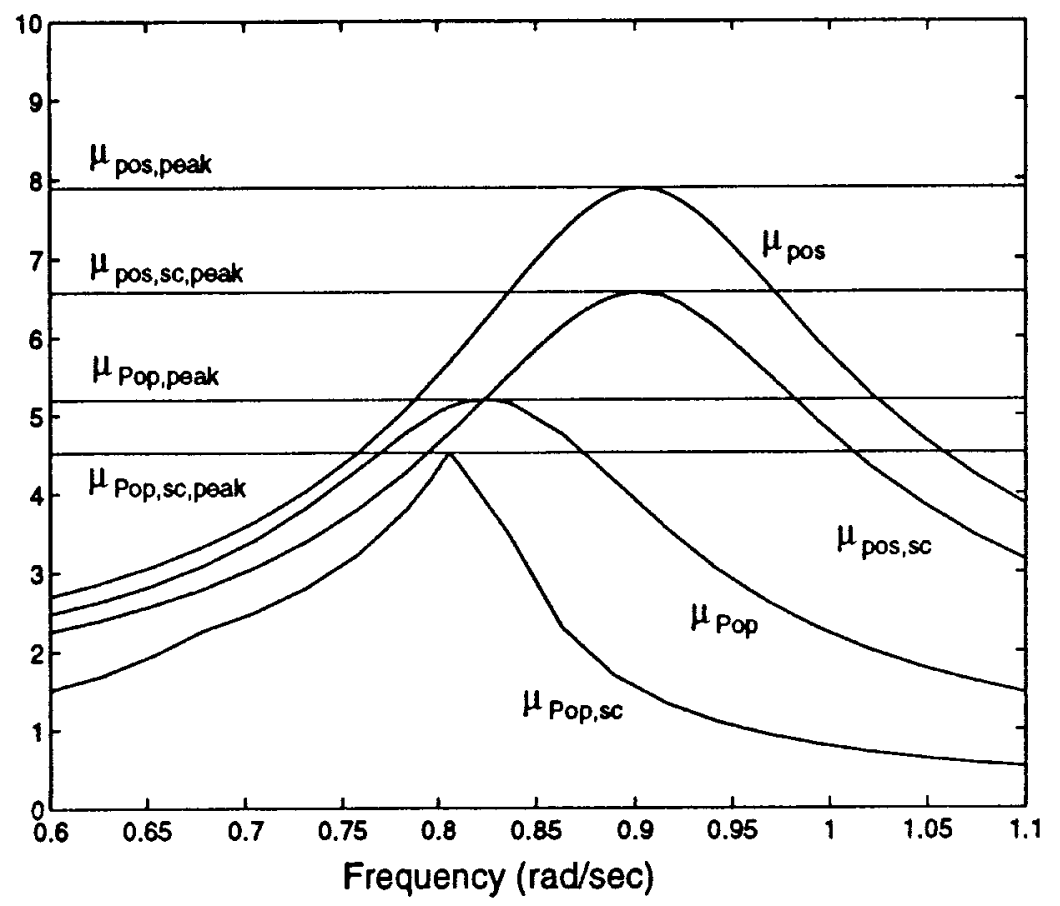

Figure 5. Frequency domain and peak upper bounds for real $\mu$ with $\Delta=\operatorname{diag}\left(\delta_{1}, \delta_{2}, \delta_{3}\right)$ 
to be the nominal model of an uncertain system in a negative feedback interconnection with an uncertain matrix. The state-space bounds (22) - (25) and the frequency domain bounds (18)-(21) will be compared for several different uncertainty structures.

First consider the case of non-repeated, diagonal uncertainty, that is, $\Delta=\operatorname{diag}\left(\delta_{1}, \delta_{2}, \delta_{3}\right)$. Each frequency domain bound was computed over a frequency range from 0.6 to $1 \cdot 1 \mathrm{rad} / \mathrm{sec}$ to focus on the region near the peak. Successively finer grids of frequencies were used to accurately compute the peak value. Then, the corresponding state-space bounds were computed. The frequency domain and state-space bounds are shown in Figure 5. Note that in all cases the state-space upper bounds non-conservatively predict the peaks of the frequency domain upper bounds so that equality holds in (22)-(25).

Next consider the cases of repeated uncertainty of the distinct forms $\Delta=\operatorname{diag}\left(\delta_{1}, \delta_{1}, \delta_{2}\right)$ and $\Delta=\operatorname{diag}\left(\delta_{1}, \delta_{2}, \delta_{2}\right)$. Each frequency domain bound was computed, using the particular uncertainty structure to define the structure of the matrices $Q$ and $N$, over a frequency range from $0 \cdot 6$ to $1 \cdot 1 \mathrm{rad} / \mathrm{sec}$ as before, and the corresponding state-space bounds were computed. All the bounds are shown in Figures 6 and 7. Again, the state-space bounds were computed. All the bounds are shown in Figures 6 and 7. Again, the state-space upper bounds non-conservatively predict the peaks of the frequency domain upper bounds.

Finally, to illustrate the relative ease and accuracy with which the peak upper bounds can be computed compared with a search over frequency, consider the repeated block structure $\Delta=\delta I$. The frequency domain bound $\mu_{\text {Pop }}(G(j \omega))$ was computed for 20 frequencies between $0 \cdot 1$ and $10 \mathrm{rad} / \mathrm{sec}$. Next, $\mu_{\text {Pop }}(G(j \omega))$ was computed for refined sets of frequencies, in this case at 20 frequencies between 0.54 and $0.89 \mathrm{rad} / \mathrm{sec}$ and then 20 frequency points between 0.76 and

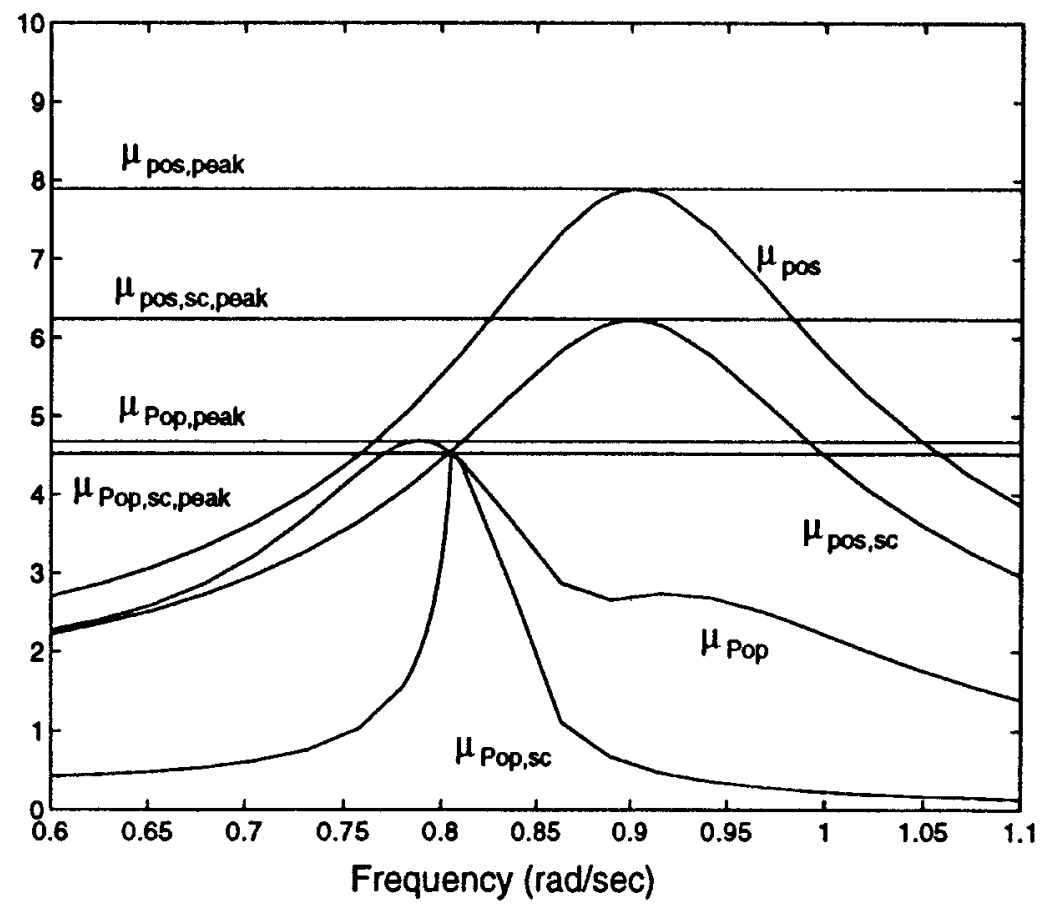

Figure 6. Frequency domain and peak upper bounds for real $\mu$ with $\Delta=\operatorname{diag}\left(\delta_{1}, \delta_{1}, \delta_{2}\right)$ 


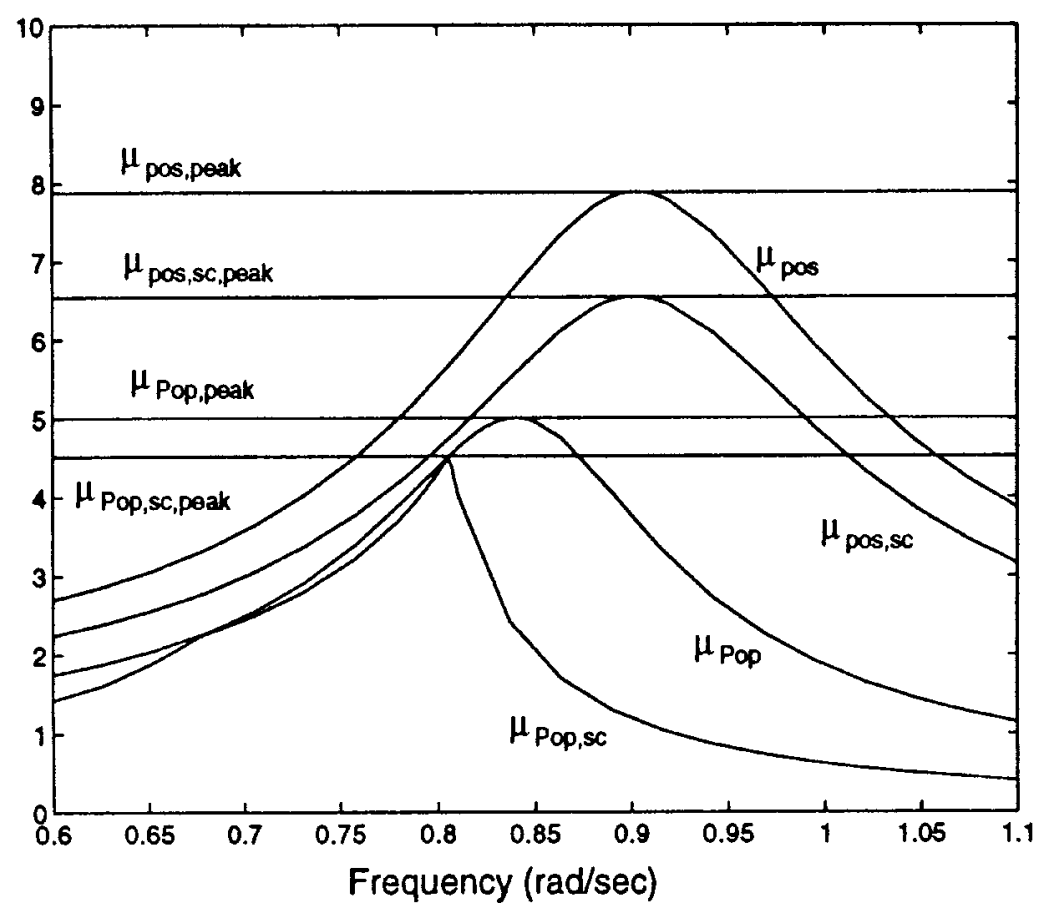

Figure 7. Frequency domain and peak upper bounds for real $\mu$ with $\Delta=\operatorname{diag}\left(\delta_{1}, \delta_{2}, \delta_{2}\right)$

$0.82 \mathrm{rad} / \mathrm{sec}$. The peak value of the third iteration is found to be $4 \cdot 2960$. The state-space bound $\mu_{\text {Pop,peak }}$ is computed for the state-space realization of the transfer function, is found to be 4.5219. Hence, the closed loop is guaranteed to be asymptotically stable for all $|\delta|<0 \cdot 2211$, the inverse of $\mu_{\text {Pop,peak. }}$. In contrast, the frequency domain bound predicts stability after three iterations for all $|\delta|<0 \cdot 2328$, the inverse of the maximum $\mu_{\text {Pop }}(G(j \omega))$ of the third iteration. In fact, the uncertainty $\delta=0.2212$ destabilizes the system. Hence, the fact that the frequency domain bound failed to capture the peak value accurately after several iterations led to erroneous conclusions about the robustness of the plant. Using sets of further refined frequencies finally results in a maximum $\mu_{\text {Pop }}(G(j \omega))$ of 4.5219 at approximately $\omega=0.8058 \mathrm{rad} / \mathrm{sec}$ after four more iterations, so that the peak bound $\mu_{\text {Pop,peak }}$ is non-conservative. Furthermore, numerical computations show the less conservative upper bound $\mu_{\mathrm{Pop}, \mathrm{sc}}(G(j \omega))$ is zero at all frequencies other than the peak frequency. Hence, as in the first example, computing the peak value using a frequency search using $\mu_{\text {Pop }, \mathrm{sc}}(G(j \omega))$ is impractical.

Finally, we consider the plant model given by ${ }^{19}$

$$
\begin{gathered}
\dot{x}=\left[\begin{array}{rrrr}
-2 & -400 & 0 \cdot 1 & 0 \cdot 2 \\
1 & 0 & 0 \cdot 5 & 0 \\
0 & 2 & -3 & -80 \\
0 & 0 & 1 & 0
\end{array}\right] x+\left[\begin{array}{ll}
2 & 0 \cdot 8 \\
0 & 0 \\
0 & 1 \\
1 & 0
\end{array}\right] u \\
y=\left[\begin{array}{llll}
1 \cdot 5 & 0 & 1 & 0 \\
0 & 1 & 2 & 2
\end{array}\right] x
\end{gathered}
$$




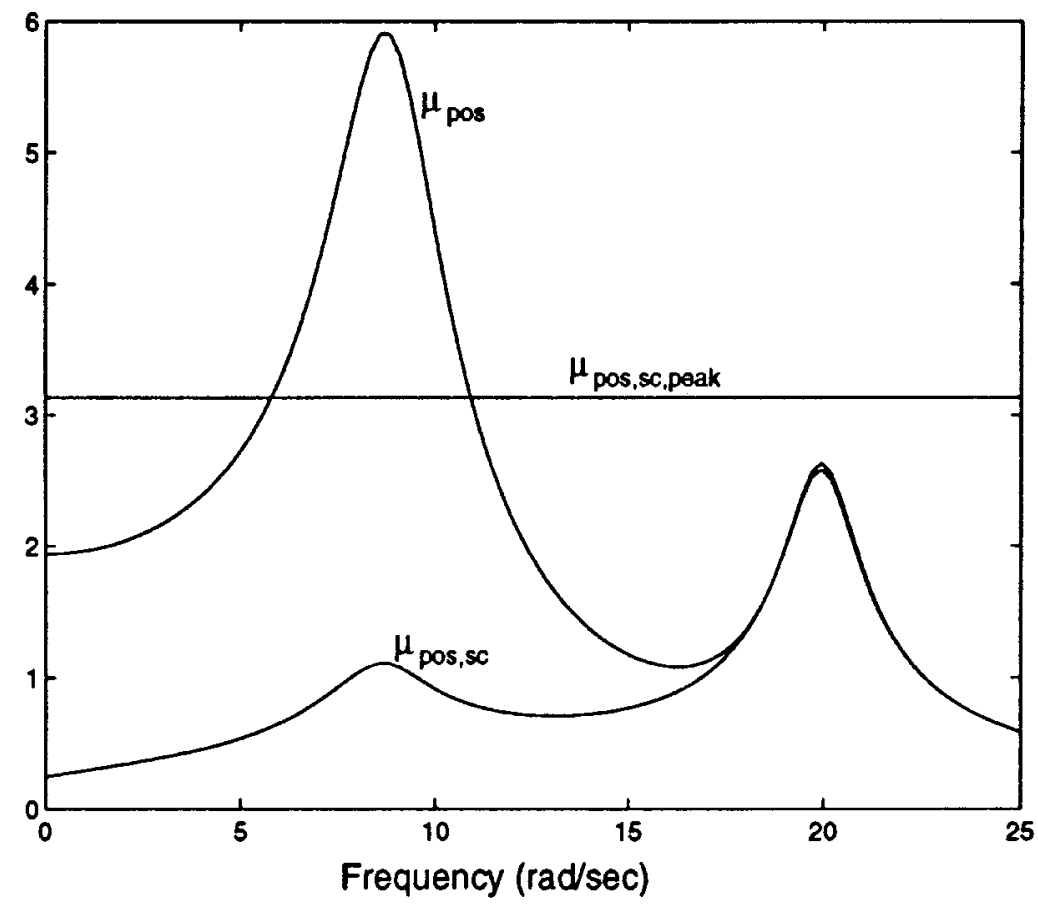

Figure 8. Upper bounds $\mu_{\text {pos }}(G(j \omega)), \mu_{\text {pos }}(G(j \omega))$ and $\mu_{\text {pos,sc,peak }}(G(j \omega))$

in a feedback interconnection with diagonal uncertainty $\Delta=\operatorname{diag}\left(\delta_{1}, \delta_{2}\right)$. The peak upper bound $\mu_{\text {pos,sc,peak }}$ was computed to be $3 \cdot 1331$ (see Figure 8), which guarantees stability for all $\Delta$ such that $\sigma_{\max }(\Delta)<0 \cdot 319$. In Reference 19 , the optimal constant $D$-scale was computed using a gradient search technique, and gave a value for the upper bound of 3.0807 , so that the system is guaranteed to be asymptotically stable for all $\Delta$ such that $\sigma_{\max }(\Delta)<0 \cdot 320$, which is slightly better than the value given by $\mu_{\text {pos,sc,peak }}$. The small discrepancy is due to computational error. Finally, the frequency-domain upper bounds $\mu_{\text {pos }}(G(j \omega))$ and $\mu_{\text {pos,sc }}(G(j \omega))$ were computed and are shown in Figure 8 . Using several refined sets of frequencies near the largest peak of $\mu_{\mathrm{pos}, \mathrm{sc}}$, its maximum value was found to be $2 \cdot 58$, so that asymptotic stability is predicted for all $\Delta$ such that $\sigma_{\max }(\Delta)<0 \cdot 390$. Hence, $\mu_{\text {pos,sc,peak }}$ is conservative for this example.

\section{CONCLUSIONS}

A procedure for computing the peak upper bounds of the structured singular value for real and complex uncertainty was presented. The positivity and Popov criteria for norm-bounded, block-structured linear uncertainty in the feedback loop were stated in terms of positive real tests. By stating these criteria for scaled transfer functions, upper bounds for the structured singular value for real and complex uncertainties can be derived. Using a generalization of the positive real lemma, the feasibility of an LMI was equated to the strict positive realness of a transfer function, so that the peak values of the frequency-domain upper bounds could be written as tests on a state-space realization of the transfer function. Such time-domain tests in the form of LMIs have the advantage that robustness bounds can be determined efficiently by solving a convex 
optimization problem, without performing an exhaustive search over frequency or using gradient search techniques.

\section{REFERENCES}

1. Packard, A. and J. Doyle, 'The complex structured singular value'. Automatica, 29(1), 71-109 (993).

2. Fan, M., A. Tit and J. Doyle, 'Robustness in the presence of mixed parametric uncertainty and unmodelled dynamics'. IEEE Trans. Automat. Control, 36(1), 25-38 (1971).

3. Young, P., M. Newlin and J. Doyle, ' $\mu$ analysis with real parameter uncertainty'. Proceedings of the IEEE Conference on Decision and Control, Brighton, UK, December 1991, pp. 1251-1256.

4. Sparks, A. G. and D. S. Bernstein, 'The scaled Popov criterion and bounds for the real structured singular value'. Proceedings of the IEEE Conference on Decision and Control, Orlando, FL, December 1994.

5. Boyd, S., L. El Ghaoui, E. Feron and V. Balakrishnan, Linear Matrix Inequalities in Systems and Control Theory, SIAM Studies in Applied Mathematics, Vol. 15, SIAM, Philadelphia, PA, 1994.

6. Gahinet, P. and A. Nemirovskii, LMI Control Tool box, The Mathworks, 1995.

7. Ly. J. H., M. G. Safonov and R. Y. Chiang, 'Real/complex multivarible stability margin computation via generalized Popov multiplier-LMI approach'. Proceedings of the American Control Conference, Baltimore, MD, 1994, pp. $425-429$.

8. Safonov, M. G. and R. Y. Chiang, 'Real/complex $K_{m}$-synthesis without curve fitting'. Control and Dynamic Systems, 56, 303-324 (1993).

9. Balakrishnan, V., Y. Huang, A. Packard and J. Doyle, 'Linear matrix inequalities in analysis with multipliers'. Proceedings of the American Control Conference, Baltimore, 1994, pp. 1228-1232.

10. Doyle, J. C. and A. Packard, 'Uncertain multivariable systems from a state space perspective'. Proceedings of the American Control Conference, 1987, Minneapolis, MN, pp. 2147-2152.

11. Sideris, A., 'Elimination of frequency search from robustness tests'. Proceedings of the IEEE Conference on Decision and Control, Honolulu, 1990, pp. 41-45.

12. Chen, C. T., Linear System Theory and Design, Saunders College Publishing, Orlando, FL, 1984.

13. Youla, D. C., 'On the factorization of rational matrices'. IRE Trans. Information Theory, 7, 172-189 (1961).

14. Ioannou, P. and G. Tao, 'Frequency domain conditions for strictly positive real transfer functions'. IEEE Trans. Automat. Control, 32(1), 53-54 (987).

15. Narendra, K. and J. Taylor, Frequency Domain Criteria for Absolute Stability, Academic Press, New York, 1973.

16. Anderson, B. D. O. and S. Vongpanitlerd, Network Analysis and Synthesis: A Modern Systems Approach, Prentice Hall, 1973.

17. Khalil, H., Nonlinear Systems, Macmillan Publishing, New York, 1992.

18. Bernstein, D. S., W. M. Haddad and A. G. Sparks, 'A Popov criterion for uncertain linear multivariable systems'. Automatica, 30, 1061-1064 (1995).

19. Haddad, W. M., E. G. Collins and R. Moser, 'Computation of the complex structured singular value using fixed-structure dynamic D-scales'. Proceedings of the IEEE Conference on Decision and Control, Orlando, FL, December 1994, pp. 3003-3008.

20. Haddad, W. M. and D. S. Bernstein, 'Robust stabilization with positive real uncertainty: beyond the small gain theorem'. Systems \& Control Letters, 17, 191-208 (1991).

21. Haddad, W. M. and D. S. Bernstein, 'Parameter dependent Lyapunov functions, constant real parameter uncertainty, and the Popov criterion in robust analysis and synthesis'. Proceedings of the IEEE Conference on Decision and Control, Brighton, UK, December 1991, pp. 2274-2279 and 2632-2633.

22. Haddad, W. M. and D. S. Bernstein, 'Explicit construction of quadratic Lyapunov functions for the small gain, positivity, circle, and Popov theorems and their application to robust stability. Part I: continuous time theory'. Int. J. Robust Nonlinear Control, 3, 313-319 (1993).

23. Packard, A., K. Zhou, P. Panley and B. Becker, 'A collection of robust control problems leading to LMI's'. Proceedings of the IEEE Conference on Decision and Control, Brighton, UK, 1991, pp. 1245-1250.

24. Tao, G. and P. Ioannou, 'Strictly positive real matrices and the Lefschez-Kalman-Yakubovich lemma'. IEEE Trans. Automat. Control, 33, 1183-1185 (1988). 